\title{
NEW RESULTS ON AUTOMORPHIC INTEGRALS AND THEIR PERIOD FUNCTIONS
}

\author{
RICHARD A. CAVALIERE
}

\begin{abstract}
Automorphic integrals, being generalizations of automorphic forms on discrete subgroups of $S L(2, \mathbf{R})$, share properties similar to those of forms. In this article I obtain a natural boundary result for integrals which is similar to that which holds for forms. If an automorphic integral on a given group behaves like a form on a subgroup of finite index (i.e., the period functions are identically zero), then in fact the integral must be a form on the whole group. Specializing to modular integrals with integer dimension I obtain a lower bound on the number of poles of the period functions which, of necessity, lie in quadratic extensions of the rationals.
\end{abstract}

I. Introduction. In recent years the concept of automorphic form has been generalized to permit an additive "period" function in the functional equation. An automorphic form $f$, on a discrete subgroup $\Gamma$ of $S L(2, \mathbf{R})$, is required to satisfy, among other conditions, the functional equation:

$$
\bar{v}(V)(c z+d)^{\delta} f(V z)=f(z), \quad V=\left(\begin{array}{cc}
a & b \\
c & d
\end{array}\right) \in \Gamma .
$$

An automorphic integral, on the other hand, is required to satisfy:

$$
\bar{v}(V)(c z+d)^{\delta} f(V z)=f(z)+\phi_{V}(z), \quad V=\left(\begin{array}{ll}
a & b \\
c & d
\end{array}\right) \in \Gamma .
$$

Here $v$ is a multiplier system on the group $\Gamma$ and the dimension $\delta$ is a real number. $\phi_{V}(z)$ is called the period function of $f$ associated with $V$. The collection $\left\{\phi_{V}(z): V \in \Gamma\right\}$ is usually constrained to lie in a suitably chosen space of functions which typically satisfy growth conditions in a fundamental region for $\Gamma$. The precise definitions appear in $\S I I$. Automorphic integrals arise naturally as integrals of forms, hence the name.

Automorphic forms have the real axis as a natural boundary. In $\S I I I$ we show that, under a necessary restriction, the same is true of automorphic integrals. In connection with automorphic integrals it is reasonable to ask if some, but not all, of the period functions can be identically zero. In $\S I V$ we show that, under certain conditions, if the period functions on a subgroup of $\Gamma$ of finite index are identically zero, then the period functions vanish on the entire group.

In [4], Knopp considered automorphic integrals of integer dimension with rational period functions on the modular group - modular integrals. He found that

Received by the editors June 16, 1986.

1980 Mathematics Subject Classification (1985 Revision). Primary 11F12, 11F11; Secondary $11 \mathrm{~F} 30$. 
the poles of the period functions must lie in quadratic extensions of the rational numbers. For these types of period functions we find, in $\S \mathrm{V}$, necessary conditions on $\delta$ in terms of the period functions and obtain a lower bound on the number of poles of rational period functions in terms of the quadratic extension in which they lie.

\section{Preliminaries.}

1. We shall confine our attention to certain discrete subgroups $\Gamma$ of $S L(2, \mathbf{R})$. We assume $-I \in \Gamma$. An element $V=\left(\begin{array}{ll}a & b \\ c & d\end{array}\right)$ of $\Gamma$ induces a linear fractional transformation of $H$, the upper half-plane, by $z \rightarrow(a z+b) /(c z+d)$. Hence, $\Gamma$ can be thought of as a group of linear, fractional transformations provided we identify $\pm\left(\begin{array}{ll}a & b \\ c & d\end{array}\right)$ with the same transformation. Specifically, we shall be interested in $H$-groups. A subgroup $\Gamma$ of $S L(2, \mathbf{R})$ is an $H$-group if:

1. $\Gamma$ is finitely generated.

2. $\Gamma$ is discrete but discontinuous at no point of $\mathbf{R}$.

3. $\Gamma$ contains translations.

As a consequence, an $H$-group has a fundamental region in $H$ with finitely many sides (see [6] for details). The vertices of the fundamental region which lie on the extended real axis are called cusps of the fundamental region or cusps of $\Gamma$. They are necessarily parabolic fixed points and conversely any parabolic fixed point of $\Gamma$ is a cusp of some fundamental region for $\Gamma$.

2. Cusp width. It will be necessary to obtain expansions of certain functions at cusps of the fundamental region. These expansions involve the notion of "cusp width" for $\Gamma$ which we now define.

DEFINITION. Let $q$ be a cusp of some fundamental region $R$ for an $H$-group $\Gamma$. If $q=\infty$, then $\Gamma_{\infty}=\langle Q\rangle$ where $Q=\left(\begin{array}{ll}1 & \lambda \\ 0 & 1\end{array}\right), \lambda>0$, and $\Gamma_{\infty}=\{V \in \Gamma \mid$ $\left.V_{\infty}=\infty\right\}$-the stabilizer of $\infty$. The width of the cusp $\infty$ is defined to be $\lambda$. For a finite cusp $q$ with stabilizer $\Gamma_{q}=\left\langle Q=\left(\begin{array}{cc}\alpha & \beta \\ \gamma & \delta\end{array}\right)\right\rangle$ we write $Q$ in its normal form $Q: 1 /\left(\tau^{\prime}-q\right)=1 /(\tau-q) \pm \gamma$. Here $\tau^{\prime}=Q \tau$ and the sign before $\gamma$ is that of $\alpha+\delta$. Since $-I \in \Gamma$ we may, without loss of generality, assume that $\gamma>0$ and $\alpha+\delta>0$. With $Q$ chosen in this way, we define the width of $q$ to be $\gamma$.

REMARK. Our definition of cusp width is not the "usual" one. However, it is equivalent (see [5, p. 269]).

We now show how the widths of distinct cusps, equivalent under $\Gamma$, are related.

PROPOSITION 1. Let $q$ be a cusp of the fundamental region $R$ with width $\lambda$. For $V=\left(\begin{array}{ll}a & b \\ c & d\end{array}\right) \in \Gamma, V q=\hat{q}$ is again a cusp of $\Gamma$. Let $\hat{\lambda}$ denote the width of $\hat{q}$. If $q$ and $\hat{q}$ are both finite, then $\hat{\lambda}=(c q+d)^{2} \lambda$. If $q=\infty$ and $\hat{q} \neq \infty$, then $\hat{\lambda}=c^{2} \lambda$.

Proof. Assume $q$ and $\hat{q}$ are finite. $\Gamma_{q}=\left\langle Q=\left(\begin{array}{cc}\alpha & \beta \\ \gamma & \delta\end{array}\right)\right\rangle, \gamma>0, \alpha+\delta>0$, and $\lambda=\gamma$. Since $\Gamma_{\hat{q}}=\left\langle V Q V^{-1}\right\rangle$, we need the matrix form of $V Q V^{-1}$ to compute $\hat{\lambda}$.

$$
V Q V^{-1}=\left(\begin{array}{cc}
* & * \\
c(\alpha d-\beta c)+d(\gamma d-c \delta) & *
\end{array}\right)
$$

and $\operatorname{Trace}\left(V Q V^{-1}\right)=\operatorname{Trace}(Q)>0$. If $c(\alpha d-\beta c)+d(\gamma d-c \delta)>0$, then $\hat{\lambda}=$ $c(\alpha d-\beta c)+d(\gamma d-c \delta)$. We will show that $c(\alpha d-\beta c)+d(\gamma d-c \delta)=\gamma(c q+d)^{2}>0$. 
Since $Q q=q$ and $Q$ is parabolic, we find $q=(\alpha-\delta) / 2 \gamma$ with the result that

$$
\begin{aligned}
\gamma(c q+d)^{2} & =c^{2} \frac{(\alpha-\delta)^{2}}{4 \gamma}+\alpha d c-\delta d c+\gamma d^{2} \\
& =c\left[\alpha d+\frac{c(\alpha-\delta)^{2}}{4 \gamma}\right]+d(d \gamma-\delta c) .
\end{aligned}
$$

It remains to show that $c(\alpha-\delta)^{2} / 4 \gamma=-\beta c$. Since $(\alpha+\delta)^{2}=4$ it follows that $(\alpha-\delta)^{2}=-4 \beta \gamma$ and the first statement of the proposition is proved. The second statement is proved by calculating a generator of the stabilizer $\Gamma_{\hat{q}}$.

3. Multiplier systems.

DEFINITION. We call a function $v$ a multiplier system of dimension $\delta \in \mathbf{R}$ for $\Gamma$ provided:

1. $v: \Gamma \rightarrow\{|z|=1\}$.

2. If $M_{i}=\left(\begin{array}{cc}* & * \\ c_{i} & d_{i}\end{array}\right) \in \Gamma$ and $M_{3}=M_{1} M_{2}$, then

$$
\bar{v}\left(M_{3}\right)\left[M_{3}: z\right]^{\delta}=\bar{v}\left(M_{1}\right) \bar{v}\left(M_{2}\right)\left[M_{1}: M_{2} z\right]^{\delta}\left[M_{2}: z\right]^{\delta}
$$

where $\left[M_{i}: z\right]=c_{i} z+d_{i}$ and "-_" denotes complex conjugation.

REMARKS. (i) We fix the branch of the many-valued function $(c z+d)^{\delta}$ by requiring, for $z \in \mathbf{C}$,

$$
z^{\delta}=|z|^{\delta} e^{i \delta \arg (z)} \quad \text { where }-\pi \leq \arg (z)<\pi .
$$

In particular $\arg (-1)=-\pi$.

(ii) When $\delta \in \mathbf{Z}$, then part 2 of the definition shows that $\bar{v}$ is a character on the matrix group $\Gamma$.

(iii) $\bar{v}$ is a function on the matrix group since $\bar{v}(I)=1$ and $\bar{v}(-I)^{2}=e^{2 \pi i \delta} \neq$ 1 unless $\delta \in \mathbf{Z}$. Thus in general, $\bar{v}(-M) \neq \bar{v}(M)$ even though these matrices correspond to the same transformation of $z$.

(iv) When $\delta \in \mathbf{Z}$, the existence of multipliers is easily demonstrated. (See $[\mathbf{5}, \mathbf{p}$. 268].) Petersson [7] has shown that multipliers exist for arbitrary groups and all dimensions.

(v) For a given multiplier system $v$ and dimension $\delta$ we define the stroke operator ||$_{v}^{\delta}$ by $\left.f\right|_{v} ^{\delta} V=\bar{v}(V)[V: z]^{\delta} f(V z)$ for any $V \in \Gamma$ and function $f$. When no confusion can occur we will suppress the multiplier and the dimension, writing $f{ }_{v}^{\delta} V=f \mid V$. We observe that $f|V W=(f \mid V)| W$ and $(f+g)|V=f| V+g \mid V$.

4. Cocycles. Let $P$ be the space of functions $g(z)$ holomorphic in $H$ such that there exist positive constants $k, \rho, \sigma$ depending on $g$ satisfying $|g(z)|<k\left(|z|^{\rho}+y^{-\sigma}\right)$ with $y=\operatorname{Im}(z)>0$. For $\Gamma$ an $H$-group and $v$ a multiplier system of dimension $\delta$ on $\Gamma$, we call a collection of functions $\left\{\phi_{V} \in P, V \in \Gamma\right\}$ a cocycle of dimension $\delta$ with multiplier system $v$ if for $V, W \in \Gamma, \phi_{V W}=\phi_{V} \mid W+\phi_{W}$. A cocycle $\left\{\phi_{V}\right\}$ is called a coboundary if there exists a $\psi \in P$ such that $\phi_{V}=\psi \mid V-\psi$.

For $\Gamma$ an $H$-group, fix a fundamental region $R$ containing $\infty$. Denote the inequivalent cusps of $R$ by $q_{0}=\infty, q_{1}, \ldots, q_{l}$ and let the stabilizer $\Gamma_{q_{i}}$ of $q_{i}$ be generated by $Q_{i}$ for $0 \leq i \leq l$. The cocycle $\left\{\phi_{V}\right\}$ is called a parabolic cocycle if for each $i$, $0 \leq i \leq l, \phi_{Q_{i}}=\psi_{i} \mid Q_{i}-\psi_{i}$ with $\psi_{i} \in P$.

REMARK. In [2] it is shown that every cocycle in $P$ is parabolic.

5. Automorphic integrals. Let $\Gamma$ be an $H$-group with $l$ finite cusps $q_{i}, 1 \leq i \leq l$, and the cusp $q_{0}=\infty$ for a particular fundamental region $R$ of $\Gamma$. Furthermore, 
assume $v$ is a multiplier system of dimension $\delta$ and $f$ is a function, meromorphic in $H$, satisfying:

1. $f \mid{ }_{v}^{\delta} V-f=\phi_{V} \in P, V \in \Gamma$.

2. For each $i, 1 \leq i \leq l,\left(\tau-q_{i}\right)^{-\delta}\left(f(\tau)-\psi_{i}(\tau)\right)$ has a limit as $\tau \rightarrow q_{i}$ within $R$. Here $\psi_{i}$ satisfies the parabolic cocycle condition at the cusp $q_{i}$.

3. $f(\tau)-\psi_{0}(\tau)$ has a limit as $\tau \rightarrow \infty$ from within $R$. Here $\psi_{0}$ satisfies the parabolic cocycle condition at the cusp $q_{0}=\infty$.

$f$ is called an automorphic integral of dimension $\delta$ with multiplier system $v$ on $\Gamma$. The collection of all such integrals is denoted by $\{\Gamma, \delta, v\} \int$.

REMARKS. (i) In 2 and 3 we permit the limit $\infty$.

(ii) 2 and 3 insure that the expansions of an automorphic integral at the cusps are left finite.

(iii) An automorphic integral can have only a finite number of poles in $R$. As a result, an automorphic integral $f$ has the following expansions near the cusps of $R$ :

$$
f(\tau)=\psi_{j}(\tau)+\left(\tau-q_{j}\right)^{\delta} \sum_{n=\mu_{j}}^{\infty} a_{n}(j) \exp \left(\frac{-2 \pi i\left(n+\kappa_{j}\right)}{\lambda_{j}\left(\tau-q_{j}\right)}\right), \quad \tau \in R,
$$

at the cusp $q_{j}$ where $0 \leq \kappa_{j}<1$ is defined by $v\left(Q_{j}\right)=\exp \left(2 \pi i \kappa_{j}\right)$ and $\lambda_{j}=$ width of $q_{j}$.

$$
f(\tau)=\psi_{0}(\tau)+\sum_{n=\mu_{0}}^{\infty} a_{n} \exp \left(\frac{2 \pi i\left(n+\kappa_{0}\right)}{\lambda_{0}}\right), \quad \tau \in R,
$$

at the cusp $q_{0}=\infty$ where $0 \leq \kappa_{0}<1$ is defined by $v\left(Q_{0}\right)=\exp \left(2 \pi i \kappa_{0}\right)$ and $\lambda_{0}=$ width of $\infty$.

REMARK. If $\mu_{j}+\kappa_{j} \geq 0$ in (2) for all $j, \mu_{0}+\kappa_{0} \geq 0$ in (3) and $f$ is holomorphic in $H$, then we call $f$ an entire automorphic integral and write $f \in C^{+}\{\Gamma, \delta, v\} \int$. If $\mu_{j}+\kappa_{j}>0$ in (2) for all $j, \mu_{0}+\kappa_{0}>0$ in (3) and $f \in C^{+}\{\Gamma, \delta, v\}$, then we write $f \in C^{0}\{\Gamma, \delta, v\}$. The set of automorphic integrals with identically zero period functions is denoted by $\{\Gamma, \delta, v\}$.

III. A natural boundary result. In order to establish the real axis as a natural boundary for certain types of automorphic integrals we require the expansions of these integrals at a dense subset of the real line. The cusps of the group provide such a set. Specifically, if $f$ is an automorphic integral on an $H$-group $\Gamma$, then $f$ has an expansion of a certain type "near" the cusps of a fundamental region. By "near" we mean in a sufficiently small parabolic sector at the cusp. The expansions of $f$ at equivalent cusps are intimately related. This relationship is explained in the following theorem, whose proof can be found in $[\mathbf{1}]$.

THEOREM 2. If $f \in\{\Gamma, \delta, v\} \int$ has expansions (2) and (3), then at the cusp $\hat{q}_{j}=V q_{j}, V=\left(\begin{array}{ll}a & b \\ c & d\end{array}\right)$, $f$ has the expansions

$$
f(\tau)=\hat{\psi}_{j}(\tau)+\left(\tau-\hat{q}_{j}\right)^{\delta} \sum_{n=\mu_{j}}^{\infty} \alpha_{n}(j) \exp \left(\frac{-2 \pi i\left(n+\kappa_{j}\right)}{\hat{\lambda}_{j}\left(\tau-\hat{q}_{j}\right)}\right) \quad \text { if } \hat{q}_{j} \neq \infty
$$


and

$$
f(\tau)=\hat{\psi}_{j}(\tau)+\sum_{n=\mu_{j}}^{\infty} \beta_{n}(j) \exp \left(\frac{2 \pi i\left(n+\kappa_{j}\right) \tau}{\hat{\lambda}_{j}}\right) \quad \text { if } \hat{q}_{j}=\infty .
$$

REMARKS. 1. $\hat{\lambda}_{j}$ is the width of the cusp $\hat{q}_{j}$ discussed in Proposition 1 .

2. $\hat{\psi}_{j}$ satisfies the parabolic cocycle condition at the cusp $\hat{q}_{j}$ and in fact $\hat{\psi}_{j}=$ $\psi_{j} \mid V^{-1}-\phi_{V-1}$.

3. In particular, we see that the lower index of summation remains the same in the expansions at equivalent cusps.

The proof of the natural boundary result will depend on an estimate of

$$
\left|\exp \left(\frac{-2 \pi i}{\lambda_{j}\left(\tau-q_{j}\right)}\right)\right| \quad \text { as } \tau \rightarrow q_{j}
$$

within a fundamental region $R$. Any such approach will eventually lie within a Stolz angle at $q_{j}: y>\left|x-q_{j}\right|$ :

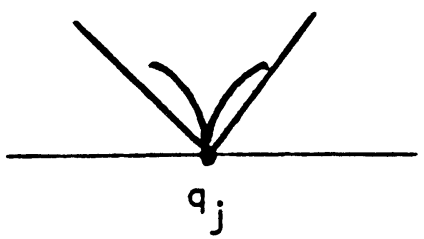

For this approach we have, at the finite cusp $q_{j}$,

$$
\begin{aligned}
\left|\exp \left(\frac{-2 \pi i}{\lambda_{j}\left(\tau-q_{j}\right)}\right)\right| & =\exp \left(\frac{-2 \pi y}{\lambda_{j}\left[\left(x-q_{j}\right)^{2}+y^{2}\right]}\right) \\
& <\exp \left(\frac{-\pi}{\lambda_{j} y}\right) \rightarrow 0 \text { as } \tau \rightarrow q_{j}
\end{aligned}
$$

At the cusp $\infty$ we have

$$
\left|\exp \left(\frac{2 \pi i \tau}{\lambda}\right)\right|=\exp \left(\frac{-2 \pi y}{\lambda}\right) \rightarrow 0 \quad \text { as } \tau \rightarrow \infty
$$

In the expansions of an automorphic integral (2) and (3) we say $f$ is holomorphic at the cusp $q_{j}$ if $\mu_{j}+\kappa_{j} \geq 0$. Here we mean holomorphic in the local uniformizing variable $t=\exp \left(-2 \pi i / \lambda_{j}\left(\tau-q_{j}\right)\right)$ or $t=\exp \left(2 \pi i \tau / \lambda_{0}\right)$ for the cusp $\infty$.

THEOREM 3. Let $f \in\{\Gamma, \delta, v\} \int$. If there is a cusp where $f$ is not holomorphic, then $f$ has the real axis as a natural boundary.

PROOF. We may without loss of generality assume that $q_{j}$ is a finite cusp. First we will show that $f$ is not analytic at $q_{j}$ as a function of the variable $\tau$. The hypotheses imply

$$
f(\tau)=\psi_{j}(\tau)+\left(\tau-q_{j}\right)^{\delta} \sum_{n=\mu_{j}}^{\infty} a_{n}(j) \exp \left(\frac{-2 \pi i\left(n+\kappa_{j}\right)}{\lambda_{j}\left(\tau-q_{j}\right)}\right) \quad \text { and } \quad \mu_{j}<0 .
$$

We have

$$
\begin{aligned}
|f(\tau)| & +\left|\psi_{j}(\tau)\right| \geq\left|f(\tau)-\psi_{j}(\tau)\right| \\
& =\left|\left(\tau-q_{j}\right)^{\delta} \exp \left(\frac{-2 \pi i\left(\mu_{j}+\kappa_{j}\right)}{\lambda_{j}\left(\tau-q_{j}\right)}\right) \sum_{n=\mu_{j}}^{\infty} a_{n}(j) \exp \left(\frac{-2 \pi i\left(n-\mu_{j}\right)}{\lambda_{j}\left(\tau-q_{j}\right)}\right)\right| .
\end{aligned}
$$


Within a Stolz angle

$$
\left|\sum_{n=\mu_{j}}^{\infty} a_{n}(j) \exp \left(\frac{-2 \pi i\left(n-\mu_{j}\right)}{\lambda_{j}\left(\tau-q_{j}\right)}\right)\right|
$$

is bounded away from 0 , since if

$$
t=\exp \left(\frac{-2 \pi i}{\lambda_{j}\left(\tau-q_{j}\right)}\right) \text { and } g(t)=\sum_{n=\mu_{j}}^{\infty} a_{n}(j) t^{n-\mu_{j}},
$$

then $g$ is continuous at $t=0$ and $g(0)=a_{\mu_{j}}(j) \neq 0$. Thus

$$
|f(\tau)|+\left|\psi_{j}(\tau)\right| \geq K\left|\tau-q_{j}\right|^{\delta} \exp \left(\frac{-\pi\left(\mu_{j}+\kappa_{j}\right)}{\lambda_{j} y}\right) \quad \text { and } \quad \mu_{j}+\kappa_{j}<0 \text {. }
$$

Here we have used (4). We now have

$$
|f(\tau)| \geq-\left|\psi_{j}(\tau)\right|+K\left|\tau-q_{j}\right|^{\delta} \exp \left(\frac{-\pi\left(\mu_{j}+\kappa_{j}\right)}{\lambda_{j} y}\right) .
$$

Since $\psi_{j} \in P$, the second term of the right side of (5) dominates and $\lim _{\tau \rightarrow q_{j}}|f(\tau)|=$ $\infty$. It follows that $f$ is not analytic, in the variable $\tau$, at $q_{j}$. Theorem 2 implies that $f$ is not analytic, in the variable $\tau$, at any cusp equivalent to $q_{j}$. However, the orbit $\Gamma q_{j}$ is dense in the real line by a well-known property of discontinuous groups. It follows that $f$ has the real axis as a natural boundary. The proof is complete.

REMARK. The hypothesis that $f$ is not holomorphic at some cusp is a necessary one, as the function $f(z)=\exp \left(2 \pi i z / \lambda_{0}\right)$ shows.

IV. A subgroup of finite index result. It may be possible for some of the period functions of an automorphic integral to be identically zero. However, we show below that for integrals with nonzero integer dimension and rational period functions, if the period functions corresponding to a subgroup of finite index vanish, then all period functions vanish.

DEFINITION. A real discrete group $\Gamma$ is called nonelementary if the limit set of $\Gamma$ contains more than two points.

REMARK. Any real discrete nonelementary group containing translations contains hyperbolic elements.

PROPOSITION 4. Let $\Gamma$ be a real discrete nonelementary group containing translations. If $\Gamma$ contains a subgroup $\hat{\Gamma}$ of finite index, $[\Gamma: \hat{\Gamma}]=n$, then $\Gamma$ has a hyperbolic decomposition modulo $\hat{\Gamma}$. Specifically $\Gamma=\bigcup_{i=1}^{n} \hat{\Gamma} A_{i}$ where the $A_{i}$ are hyperbolic.

ProOF. The hypotheses imply $\Gamma=\bigcup_{i=1}^{n} \hat{\Gamma} B_{i}$ where $B_{i} \in \Gamma$. Using the preceding remark we may without loss of generality assume that $\Gamma=\bigcup_{i=1}^{n} \hat{\Gamma} B_{i}$, where $B_{i}=\left(\begin{array}{cc}\alpha_{i} & \beta_{i} \\ \gamma_{i} & \delta_{i}\end{array}\right)$ and $\gamma_{i} \neq 0$. We will show that a hyperbolic $A_{i} \in \Gamma$ can be chosen such that $\hat{\Gamma} B_{i}=\hat{\Gamma} A_{i}$. Let $S=\left(\begin{array}{ll}1 & \lambda \\ 0 & 1\end{array}\right) \in \Gamma$. There exists a positive integer $m$ such thai $S^{m} \in \hat{\Gamma}$. For any $\sigma \in \mathbf{Z}$, we have

$$
S^{\sigma m} B_{i}=\left(\begin{array}{cc}
\alpha_{i}+\sigma m \lambda \gamma_{i} & \beta_{i}+\sigma m \lambda \delta_{i} \\
\gamma_{i} & \delta_{i}
\end{array}\right)
$$


Hence Trace $\left(S^{\sigma m} B_{i}\right)=\operatorname{Trace}\left(B_{i}\right)+\sigma m \lambda \gamma_{i}$ and we can choose $\sigma=\sigma_{i}$ such that Trace $\left(B_{i}\right)+\sigma_{i} m \lambda \gamma_{i}>2$. For this choice of $\sigma_{i}, S^{\sigma_{i} m} B_{i}$ will be hyperbolic and $\hat{\Gamma} B_{i}=\hat{\Gamma} S^{\sigma_{i} m} B_{i}$. Putting $A_{i}=S^{\sigma_{i} m} B_{i}$ we have the statement of the proposition.

REMARK. There is an infinite number of choices of $\sigma$ for each $i$. This observation leads to the following lemma, which we state without proof.

LEMMA 5. If $F$ is a finite subset of the plane, then the $A_{i}$ whose existence is quaranteed by Proposition 4 can be chosen such that the fixed points of $A_{i}$ do not belong to $F$.

We are now able to prove

THEOREM 6. Let $\Gamma$ be a real, discrete, nonelementary group containing translations such that

1. $\Gamma$ contains a subgroup $\hat{\Gamma}$ of finite index, $[\Gamma: \hat{\Gamma}]=n$,

2. $f \in\{\Gamma, \delta, v\}$ and $f \in\{\hat{\Gamma}, \delta, v\}$,

3. $\phi_{V}=f \mid V-f$ is rational for all $v \in \Gamma$ and $\delta \neq 0$. Then $f \in\{\Gamma, \delta, v\}$.

REMARKS. 1. Theorem 6 states that an automorphic integral whose period functions vanish on a large enough subgroup is actually an automorphic form on the whole group.

2. The rationality of $\phi_{V}$ for all $V \in \Gamma$ implies $\delta \in Z$.

ProOF OF THEOREM 6. Let $W \in \Gamma$. We will show that $\phi_{W}=0$. By Proposition 4 and Lemma 5 we have $\Gamma=\bigcup_{i=1}^{n} \hat{\Gamma} A_{i}$ with $A_{i}$ hyperbolic and the fixed points of $A_{i}$ are not zeros or poles of $\phi_{W}$. For some $i, W \in \hat{\Gamma} A_{i}$ and $W=V A_{i}$, $V \in \hat{\Gamma}$. Hence $\phi_{W}=\phi_{V A_{i}}=\phi_{V} \mid A_{i}+\phi_{A_{i}}=\phi_{A_{i}}$ where we have used the second hypothesis. The first hypothesis implies the existence of a positive integer $p$ such that $A_{i}^{p} \in \hat{\Gamma}$. For any integer $j$ we have

$$
\phi_{A_{i}}=\phi_{A_{i}^{j p}}\left|A_{i}+\phi_{A_{i}}=\phi_{A_{i}^{j p+1}}=\phi_{A_{i}}\right| A_{i}^{j p}+\phi_{A_{i}^{j p}}=\phi_{A_{i}} \mid A_{i}^{j p} .
$$

Thus

$$
\phi_{A_{i}}(z)=\bar{v}\left(A_{i}^{j p}\right)\left[A_{i}^{j p}: z\right]^{\delta} \phi_{A_{i}}\left(A_{i}^{j p} z\right) .
$$

If $\phi_{A_{i}}(z)$ is a constant, then since $\delta \neq 0$ we have $\phi_{A_{i}}(z) \equiv 0$ and $\phi_{W}=0$. If $\phi_{A_{i}}(z)$ is nonconstant then $\phi_{A_{i}}$ has a finite zero or pole $z_{0}$. It follows that $\bar{v}\left(A_{i}^{j p}\right)\left[A_{i}^{j p}\right.$ : $\left.z_{0}\right]^{\delta} \phi_{A_{i}}\left(A_{i}^{j p} z_{0}\right) \in\{0, \infty\}, j \in Z$. There exists, at most, one value $\hat{j} \in Z$ such that $\left[A_{i}^{\hat{j} p}: z_{0}\right]=0$. Thus $A_{i}^{j p}\left(z_{0}\right)$ is a zero or pole for an infinite set $\{j\}$. Moreover, $\left\{A_{i}^{j p}\left(z_{0}\right)\right\}$ is an infinite set since $z_{0}$ is not a fixed point of $A_{i}$ and $A_{i}$ has infinite order as a transformation. Since $\phi_{A_{i}}(z)$ is nonconstant and rational we have obtained a contradiction. Therefore, $\phi_{A_{i}}(z)$ must be constant and, as shown above, identically zero and the theorem is proved.

REMARKS. 3. Theorem 6 remains true when $\delta=0$ as long as $v \equiv 1$.

PROOF. (6) reduces to $\phi_{A_{i}}(z)=\phi_{A_{i}}\left(A_{i}^{j p} z\right)$. If $\phi_{A_{i}}(z)$ is a constant $c$, then $c=\phi_{A_{i}^{p}}(z)=p \cdot c$ and $c=0$ if $p \neq 1$. If $p=1$, then $\phi_{A_{i}}=0$ by hypothesis 2 .

If $\phi_{A_{i}^{p}}(z)$ is nonconstant, then as in the proof above, a contradiction is obtained.

4. The following example shows that $\delta$ must be nonzero in Theorem 6 if $v \neq 1$. We take for our group the modular group $\Gamma(1)$ generated by $S=\left(\begin{array}{ll}1 & 1 \\ 0 & 1\end{array}\right)$ and $T=\left(\begin{array}{cc}0 & -1 \\ 1 & 0\end{array}\right)$. 
The commutator subgroup $\Gamma^{\prime}$ has index 6 and $\Gamma^{\prime \prime} \backslash \Gamma(1)$ is the cyclic group of order 6 generated by $\Gamma^{\prime} S$. The multiplier system, a character on $\Gamma(1)$ in this case, is specified by $\bar{v}(S)=\exp (\pi i / 3)$ and $\bar{v}(T)=-1$.

We choose $f(z) \equiv k-$ a nonzero constant, $\delta=0$, and find

$$
\phi_{S}=f \mid S-f=v(S) k-k=k(v(S)-1) .
$$

We also have

$$
\phi_{T}=f \mid T-f=v(T) k-k=k(v(T)-1)=-2 k .
$$

Applying the equation $f \mid V=f+\phi_{V}$ to $f=k$ yields $v(V) k=k+\phi_{V}$. Since $v(V)=1$ for all $V \in \Gamma^{\prime}$ we find that $\phi_{V}=0$ for all $V \in \Gamma^{\prime}$. Hence $f=k \in\left\{\Gamma^{\prime}, 0, v\right\}$ but since $\phi_{S} \neq 0$ we see that $f=k \in\{\Gamma(1), 0, v\} \int-\{\Gamma(1), 0, v\}$.

V. Modular integrals. We now confine our attention to modular integrals with rational period functions of even dimension with multiplier system identically 1. Specifically, we consider functions $f$ satisfying $(c z+d)^{-2 k} f(V z)=f(z)+\phi_{V}(z)$ where $V \in \Gamma(1)$ and $\phi_{V}$ is rational for all $V \in \Gamma(1)$. The cocycle $\left\{\phi_{V}\right\}$ is determined by $\phi_{S}$ and $\phi_{T}$ where $S$ and $T$ are the generators of $\Gamma(1)$. We make the further assumption that $\phi_{S}=0$. Hence $f(z+1)=f(z)$ and $\left\{\phi_{V}\right\}$ is determined by $\phi_{T}=\phi$ and the cocycle condition $\phi_{V W}=\phi_{V} \mid W+\phi_{W}$. We have the relations $T^{2}=(S T)^{3}=I$, and using (1), we find

$$
\begin{gathered}
z^{-2 k} \phi\left(\frac{-1}{z}\right)+\phi(z)=0 \\
(z-1)^{-2 k} \phi\left(\frac{-1}{z-1}\right)+z^{-2 k} \phi\left(\frac{z-1}{z}\right)+\phi(z)=0 .
\end{gathered}
$$

Equations (7) and (8) yield

$$
(z+1)^{-2 k} \phi\left(\frac{z}{z+1}\right)+\phi(z+1)=\phi(z) .
$$

Equations (7) and (8) are necessary for the existence of a modular integral $f \in$ $\{\Gamma(1),-2 k, 1\}$, with period function $\phi=\phi_{T}$ and $\phi_{S}=0$. A generalized Poincaré series construction shows them to be sufficient (see [2] for details). Since any two modular integrals with the same period function $\phi$ differ by a form, we conclude that the collection of "distinct" modular integrals with rational period functions is in one-to-one correspondence with the collection of rational period functions $\phi$. This observation motivates the study of the rational period functions $\phi$. We begin with some examples.

If $f \in\{\Gamma(1),-2 k, 1\}$ and $F=f^{\prime} / f$, then $F \in\{\Gamma(1),-2,1\}$ with $\phi_{V}=$ $-2 k c /(c z+d)$. Hence $\phi=\phi_{T}=-2 k / z$. In [5] Knopp constructs, for odd $k$, the function

$$
\phi_{2 k}(z)=\left(z-z_{0}\right)^{-k}\left(z+z_{0}^{\prime}\right)^{-k}+\left(z+z_{0}\right)^{-k}\left(z-z_{0}^{\prime}\right)^{-k}
$$

where $z_{0}=(1+\sqrt{5}) / 2$ and $z_{0}^{\prime}=1 / z_{0} . \phi_{2 k}$ satisfies $(7)$ and (8), hence there is a modular integral with period function $\phi_{2 k}$.

Examination of the set of zeros and poles of a rational period function has proven useful and is the point view we adopt here. In this context Knopp has proven the following theorem: 
THEOREM 7 (THEOREM 1 IN [4]). If $p$ is a finite pole of any rational period function satisfying (7) and (8), then there is a squarefree $n \in \mathbf{Z}^{+}$such that $p \in$ $Q(\sqrt{n})$. If $p \in Q$, then $p=0$.

Using (7) we may, in a straightforward manner, deduce

PROPOSITION 8. If $\phi$ is a rational function satisfying ( 7$)$ and $z_{0} \notin\{0, i, \infty\}$ is a zero (pole) of $\phi$, then $-1 / z_{0}$ is also a zero (pole).

Moreover, the orders of $z_{0}$ and $-1 / z_{0}$ are the same. The following theorem gives necessary conditions, which are easily checked, for a rational function to be a period function of some modular integral of dimension $-2 k$.

THEOREM 9. If

$$
\phi(z)=\frac{r(z)}{s(z)}=\frac{a_{n} z^{n}+\cdots+a_{n_{0}} z^{n_{0}}}{b_{m} z^{m}+\cdots+b_{m_{0}} z^{m_{0}}}
$$

is a rational function satisfying (7) and (8), then $2 k=\left(m+m_{0}\right)-\left(n+n_{0}\right)$. 0 .

REMARK. We assume $r(z)$ and $s(z)$ are relatively prime. In particular $n_{0} \cdot m_{0}=$

Proof of THEOREM 9. Equation (7) yields

$$
\frac{\phi(-1 / z)}{\phi(z)}=-z^{2 k}
$$

If $s(z)=c z^{m_{0}} \prod_{i=1}^{\hat{m}}\left(z-z_{i}\right)^{\alpha_{i}}$, then by Theorem 7 and Proposition 8 we have $z_{j} \neq 0$, $i$ and $-1 / z_{j}=z_{l}$, with $\alpha_{j}=\alpha_{l}$. Hence

$$
s\left(\frac{-1}{z}\right)=(-1)^{m_{0}} z^{-2 m_{0}-\sum_{j=0}^{\hat{m}} \alpha_{j}}\left(\prod_{j=0}\left(-z_{j}\right)^{\alpha_{j}}\right) s(z) .
$$

Similarly if $r(z)=\lambda z^{n_{0}} \prod_{j=0}^{\hat{n}}\left(z-w_{j}\right)^{\beta_{j}}$, then

$$
r\left(\frac{-1}{z}\right)=(-1)^{n_{0}} z^{-2 n_{0}-\sum_{j=0}^{\hat{n}} \beta_{j}}\left(\prod_{j=0}^{\hat{n}}\left(-w_{j}\right)^{\beta_{j}}\right) r(z)
$$

and (10) becomes

$$
(-1)^{\left(n_{0}+m_{0}\right)} \frac{\prod_{j=0}^{\hat{n}}\left(-w_{j}\right)^{\beta_{j}}}{\prod_{j=0}^{\hat{m}}\left(-z_{j}\right)^{\alpha_{j}}} \cdot z^{2 m_{0}+\sum_{j=0}^{\hat{m}} \alpha-\left(2 n_{0}+\sum_{j=0}^{\hat{n}} \beta_{j}\right)}=-z^{2 k}
$$

Since $m_{0}+\sum_{j=0}^{\hat{m}} \alpha_{j}=m$ and $n_{0}+\sum_{j=0}^{\hat{n}} \beta_{j}=n$ we find

$$
\left(m+m_{0}\right)-\left(n-n_{0}\right)=2 k \quad \text { and } \quad(-1)^{n_{0}+m_{0}} \frac{\prod_{j=0}^{\hat{n}}(-w)^{\beta_{j}}}{\prod_{j=0}^{\hat{m}}\left(-z_{j}\right)^{\alpha_{j}}}=-1 .
$$

The proof is complete.

REMARK. If we replace $z$ by $z-1$ in (9) and differentiate, then upon evaluation at $z=1$ we find $2 k=\phi^{\prime}(1) / \phi(0)$ provided $\phi(0) \notin\{0, \infty\}$.

According to Theorem 7 , the irrational poles of a rational period function lie in $Q(\sqrt{n})$ for some squarefree $n$. Given a rational period function $\phi$ with poles 
in $Q(\sqrt{n})$ we will obtain a lower bound on the number of such poles. The exact statement appears in Theorem 13.

If $p$ is a pole of $\phi$ and $p \in Q(\sqrt{n})-Q$, then by (9) $V_{1} p$ is also a pole where $V_{1}=S=\left(\begin{array}{ll}1 & 1 \\ 0 & 1\end{array}\right)$ or $V_{1}=V=\left(\begin{array}{ll}1 & 0 \\ 1 & 1\end{array}\right)$. Similarly $V_{2} V_{1} p$ is a pole where $V_{2} \in\{S, V\}$. Since $\phi$ has only a finite number of poles, there exist integers $m>l \geq 0$ such that $V_{m} V_{m-1} \cdots V_{1} p=V_{l} V_{l-1} \cdots V_{1} p$. We may assume $m$ and $l$ are the smallest integers with the stated property. Hence $\phi$ has at least $m$ poles.

If $p^{\prime}=V_{l} \cdots V_{1} p$ then $p^{\prime} \in Q(\sqrt{n})$ is a fixed point of $V_{m} V_{m-1} \cdots V_{l+1}=\left(\begin{array}{ll}a & b \\ c & d\end{array}\right)$ and we must have $(a+d)^{2}-4=j^{2} n$ for some $j \in \mathbf{Z}$. As $n$ grows so must $a+d$ and, because of the special form of the $V_{i}, l+1 \leq i \leq m$, so must the length of the factorization $V_{m} V_{m-1} \cdots V_{l+1}$. However, the length of this factorization has a direct bearing on the number of poles and a lower bound will result. In order to implement this scheme, the relationship between $V_{m} V_{m-1} \cdots V_{l+1}$ and $n$ must be determined. We begin with two lemmas.

LEMMA 10. If $r \in \mathbf{Z}^{+}$and $(S V)^{r}=\left(\begin{array}{cc}\alpha_{r} & \beta_{r} \\ \gamma_{r} & \delta_{r}\end{array}\right)$, then $\alpha_{r}=\gamma_{r}+\delta_{r}$ and $\gamma_{r}=\beta_{r}$.

The proof is by induction on $r$ (see [1] ).

LEMMA 11. If $\left(\begin{array}{ll}a_{r} & b_{r} \\ c_{r} & d_{r}\end{array}\right)=W_{2 r}(S, V)$ - a word of length $2 r$ in $S$ and $V$ with only positive exponents, then

$$
\operatorname{Max}_{W_{2(r+1)}(S, V)}\left\{a_{r+1}, b_{r+1}, c_{r+1}, d_{r+1}\right\}=\operatorname{Max}_{W_{2 r}(S, V)}\left\{a_{r}+b_{r}+c_{r}+d_{r}\right\} .
$$

ProOF. Every word $W_{2(r+1)}(S, V)$ with positive exponents has the form $W_{2(r+1)}(S, V)=V_{1} W_{2 r}(S, V) V_{2}$ with $V_{1}, V_{2} \in\{S, V\}$. Calculation of the four possibilities completes the proof (see [1]).

THEOREM 12. Let $W_{2 r}(S, V)$ be a word of length $2 r$ in $S$ and $V$ in which only positive exponents occur. If

$$
W_{2 r}(S, V)=\left(\begin{array}{ll}
a_{r} & b_{r} \\
c_{r} & d_{r}
\end{array}\right) \quad \text { and } \quad(S V)^{r}=\left(\begin{array}{ll}
\alpha_{r} & \beta_{r} \\
\gamma_{r} & \delta_{r}
\end{array}\right),
$$

then for fixed $r$

$$
a_{r}+b_{r}+c_{r}+d_{r} \leq \alpha_{r}+\beta_{r}+\gamma_{r}+\delta_{r}=\alpha_{r+1}
$$

$$
a_{r}+d_{r} \leq \alpha_{r}+\delta_{r} .
$$

The proof is by induction on $r$ and uses Lemmas 10 and 11. We are indebted to Peter Waterman and Robert Styer for the proof of Theorem 12 (see [1]). We can state our final result. 
THEOREM 13. Let $\phi(z)$ be a rational function satisfying equations (7) and (8) and such that $\phi(z)$ has a pole in $Q(\sqrt{n})-Q$ for some squarefree $n$. If $\sigma$ is the number of poles of $\phi$ in $Q(\sqrt{n})$, then

$$
\sigma>2\left(\frac{\log n-\log 4 \lambda_{2}}{\log \lambda_{2}}\right) \quad \text { where } \lambda_{2}=\frac{3+\sqrt{5}}{2} .
$$

PROOF. By the discussion preceding Lemma 10, with an obvious change in notation, we may infer the existence of a pole $z_{0}=V_{l} V_{l-1} \cdots V_{1} p$ and a positive integer $\rho=m-l$ such that $M_{\rho} M_{\rho-1} \cdots M_{1} z_{0}=z_{0}$ and $z_{0}, M_{1} z_{0}, M_{2} M_{1} z_{0}, \ldots, M_{\rho-1} M_{\rho-2}$ $\cdots M_{1} z_{0}$ are poles of $\phi$ where $M_{i}=V_{l+i} \in\{S, V\}$. If $M_{\rho} \cdots M_{1}=\left(\begin{array}{ll}a & b \\ c & d\end{array}\right)$ then

$$
z_{0}=\frac{(a-d) \pm \sqrt{(a+d)^{2}-4}}{2 c} .
$$

It follows that $(a+d)^{2}-4=j^{2} n$ with the result that $n^{1 / 2}<a+d$. Now $a+d$ is the trace of a word in $S$ and $V$ of length $\rho$ in which only positive exponents occur. If $\rho$ is even, then we may invoke Theorem $12(\mathrm{v})$ to find $a+d \leq \operatorname{Trace}\left((S V)^{\rho / 2}\right)$. If $\rho$ is odd then we have $a+d \leq \operatorname{Trace}\left((S V)^{(\rho+1) / 2}\right)$ since the trace is an increasing function of word length for the type of words considered here (all positive exponents).

The eigenvalues of $S V$ are

$$
\lambda_{1}=\frac{3-\sqrt{5}}{2} \text { and } \lambda_{2}=\frac{3+\sqrt{5}}{2} .
$$

With

$$
M=\left(\begin{array}{cc}
\frac{1-\sqrt{5}}{2} & \frac{1+\sqrt{5}}{2} \\
1 & 1
\end{array}\right)
$$

we obtain $M^{-1}(S V) M=\left(\begin{array}{cc}\lambda_{1} & 0 \\ 0 & \lambda_{2}\end{array}\right)$. We may now calculate the trace of any power of $S V$ as

$$
\operatorname{Trace}\left((S V)^{r}\right)=\operatorname{Trace}\left(M\left(\begin{array}{cc}
\lambda_{1}^{r} & 0 \\
0 & \lambda_{2}^{r}
\end{array}\right) M^{-1}\right)=\lambda_{1}^{r}+\lambda_{2}^{r}<2 \lambda_{2}^{r} .
$$

It follows that

$$
\begin{gathered}
n^{1 / 2}<a+d \leq \operatorname{Trace}\left((S V)^{\rho / 2}\right)<2 \lambda_{2}^{\rho / 2}, \quad \rho \text { even, } \\
n^{1 / 2}<a+d \leq \operatorname{Trace}\left((S V)^{(\rho+1) / 2}\right)<2 \lambda_{2}^{(\rho+1) / 2}, \quad \rho \text { odd }
\end{gathered}
$$

Hence

$$
\begin{gathered}
\rho>\frac{\log n-\log 4}{\log \lambda_{2}}, \quad \rho \text { even, } \\
\rho>\frac{\log n-\log 4}{\log \lambda_{2}}-1, \quad \rho \text { odd. }
\end{gathered}
$$

By equation (7) we may assume without loss of generality that $z_{0}>0$ and thus we have at least $\rho$ positive poles. Equation (7) implies that we have at least this many negative poles and the proof is complete.

REMARK. When $n=p$, a prime, we obtain a greater lower bound. $j^{2} p=$ $(a+d)^{2}-4=(a+d+2)(a+d-2)$ and $p-2 \leq a+d$. We find, if $p \neq 2$

$$
\frac{p-2}{2}<\lambda_{2}^{\rho / 2} \text { and } 2 \frac{\log ((p-2) / 2)}{\log \lambda_{2}}<\rho \text { if } \rho \text { is even, }
$$




$$
\frac{p-2}{2}<2 \lambda_{2}^{(\rho+1) / 2} \text { and } 2 \frac{\log ((p-2) / 4)}{\log \lambda 2}-1<\rho \text { if } \rho \text { is odd. }
$$

Hence

$$
\sigma>2\left(2 \frac{\log ((p-2) / 4)}{\log \lambda_{2}}-1\right) \text {. }
$$

\section{REFERENCES}

1. R. A. Cavaliere, Automorphic integrals and their period functions, Doctoral Dissertation, Temple University, 1984.

2. M. I. Knopp, Some new results on the Eichler cohomology of automorphic forms, Bull. Amer. Math. Soc. 80 (1974), 607-632.

3. __ Rational period functions of the modular group, Duke Math. J. 45 (1978), 47-62.

4. _ Rational functions of the modular group. II, Glasgow Math. J. 22 (1981), 185-197.

5. J. Lehner, Discontinuous groups and automorphic functions, Math. Surveys, no. 8, Amer. Math. Soc., Providence, R.I., 1964.

6. 1966.

7. H. Petersson, Theorie der automorphen Formen beliebiegen reeler Dimension und ihre Darstellung durch eine neue Art Poincareschen Reihen, Math. Ann. 103 (1930), 369-436.

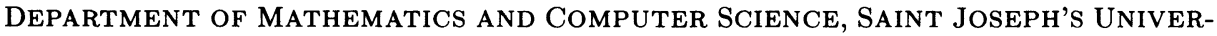
Sity, 5600 City AVenue, Philadelphia, PEnNSylvania 19151 